\title{
EVALUACIÓN DEL SERVICIO EDUCATIVO COMO INSTRUMENTO FACILITADOR DEL DESARROLLO PERSONAL DE PERSONAS CON AUTISMO
}

\section{EVALUATION OF THE EDUCATIONAL SERVICE AS AN INSTRUMENT TO FACILITATE THE DEVELOPMENT OF PEOPLE WITH AUTISM}

\author{
Alejandro Ros-Gálvez ${ }^{1}$. Universidad Católica de Murcia. España. \\ argalvez@ucam.edu
}

María Concepción Parra-Meroño². Universidad Católica de Murcia. España. mcparra@ucam.edu

José Manuel Santos-Jaén ${ }^{3}$. Universidad Católica de Murcia. España.

imsantos@ucam.edu

\section{RESUMEN}

La apuesta por el desarrollo personal y mejora de la calidad de vida de las personas con discapacidad intelectual y sus familias implica necesariamente prestar unos servicios de calidad que satisfagan sus necesidades. El objetivo de este trabajo es evaluar el Servicio de Apoyo Educativo que presta ASTRADE a niños con Trastorno del Espectro Autista (TEA). Para ello se ha realizado una encuesta a los padres o tutores de los niños en la que se les pide que evalúen la calidad del servicio recibido y que expresen su grado de satisfacción con el mismo. Los resultados indican que los usuarios están muy satisfechos con el servicio y que perciben un alto grado de calidad del mismo. Este trabajo demuestra que la Asociación ha conseguido transmitir, como uno de sus valores, la gran implicación, atención personal, confianza y amabilidad de sus profesionales hacia las personas con TEA y su entorno.

PALABRAS CLAVE: calidad del servicio, satisfacción del usuario, SERVQUAL, autismo, desarrollo personal, TEA, intervención educativa.

\section{ABSTRACT}

The focus on personal development and improvement of the quality of life of people with intellectual disabilities and their families implies necessarily to provide quality

\footnotetext{
1 Alejandro Ros-Gálvez: Universidad Católica de Murcia. Departamento de Ciencias Sociales, Jurídicas y de la Empresa. Murcia, España.

2 María Concepción Parra-Meroño: Universidad Católica de Murcia. Departamento de Ciencias Sociales, Jurídicas y de la Empresa, Murcia, España.

3 José Manuel Santos-Jaén: Universidad Católica de Murcia. Departamento de Ciencias Sociales, Jurídicas y de la Empresa. Murcia, España.
} 
services that meet their needs. The aim of this work is to assess the Educational Support Service offered by ASTRADE to children with Autism Spectrum Disorder (ASD). For this reason, we performed a survey to their parents or guardians and we asked them both to assess the quality of the perceived service and their extent of satisfaction with it. The results suggest that users are very satisfied with this service and perceive a high extent of quality. This paper also demonstrates that the Association transmits, as one of its values, the great implication, personal attention, confidence and kindness of their professionals to the people with ASD and their environment.

KEY WORDS: service quality, user satisfaction, SERVQUAL, autism, personal development, TEA, educational intervention.

\section{Cómo citar el artículo:}

Ros-Gálvez, A., Parra-Meroño M. C. y Santos-Jaén, J. M. (2016). Evaluación del servicio educativo como instrumento facilitador del desarrollo personal de personas con autismo. Revista de Ciencias de la Comunicación e Información, 21(2), 21-33.

doi: http://doi.org/10.35742/rcci.2016.21(2).21-33

\section{INTRODUCCIÓN}

Las organizaciones que integran el Tercer Sector están dirigidas a mejorar la calidad de vida de las personas a las cuales prestan sus servicios, extendiéndose a sus familiares cuando su público objetivo son personas con discapacidad intelectual (Barranco-Expósito, 2002; García-Dávila y Crespo-Cuadrado, 2008; Cuesta-Gómez, Casado-Muñoz y Lezcano-Barbero, 2010). Esta calidad de vida pasa por conseguir un adecuado desarrollo personal del individuo con discapacidad y permitir su participación efectiva y con pleno derecho en la sociedad actual.

En las últimas décadas el esfuerzo ingente de las asociaciones de familiares, junto con las ayudas públicas y el imprescindible trabajo desarrollado por los profesionales del sector, han permitido mejorar sensiblemente la calidad de vida de este colectivo, pasando de actuaciones segregadoras a un objetivo, ya no solo integrador, sino también inclusivo, de las personas con discapacidad intelectual en la sociedad (Tamarit-Cuadrado, 2005; Crosso, 2010). Se produce por tanto un giro de ciento ochenta grados en los modelos de atención, obteniéndose resultados positivos en aquellas áreas que inciden en la calidad de vida (Schalock y Verdugo-Alonso, 2007; Tamarit-Cuadrado, 2005): desarrollo personal, autodeterminación, relaciones interpersonales, inclusión social, derechos, bienestar emocional, bienestar físico y bienestar material. Así, el logro del desarrollo personal de las personas con discapacidad constituye uno de los principios básicos de estas organizaciones, con la finalidad de mejorar su calidad de vida.

Entre los distintos trastornos que afectan al desarrollo personal se encuentra el Trastorno del Espectro Autista (TEA), observándose un alto grado de variabilidad en las personas que lo padecen (Artigas, 1999; Millá y Mulas, 2009) y el 
crecimiento exponencial de los casos diagnosticados en los últimos años (ForteaSevilla, Escandell-Bermúdez y Castro-Sánchez). El TEA afecta en la inmensa mayoría de los casos al desarrollo personal de aquellos que lo padecen, al afectar a las áreas de relaciones sociales, lenguaje y comunicación, flexibilidad mental y comportamental, y ficción e imaginación (Rivière, 2002). Además, al manifestarse en la mayoría de los casos en edades tempranas, se les ha de posibilitar el acceso a una educación adecuada y de calidad, pilar clave para el desarrollo personal de cualquier individuo con o sin discapacidad. De hecho, la Convención de la ONU (Giné y Durán, 2010) establece el derecho de las personas con discapacidad al acceso a una educación inclusiva y de calidad que les posibilite su desarrollo a nivel personal. Ahora bien, la consecución de la inclusión educativa implica necesariamente que el servicio educativo se ajuste a las necesidades específicas de cada individuo, así como también facilitarles las medidas de apoyo necesarias que les permitan alcanzar el máximo desarrollo social y personal, en favor de una sociedad más humana y justa.

La Asociación ASTRADE ${ }^{4}$-en colaboración con FEAPS Región de Murcia ${ }^{5}$ y la Consejería de Educación, Formación y Empleo de la CARM- hace posible este compromiso en la Comunidad Autónoma de la Región de Murcia (CARM) a través de su Servicio de Apoyo Educativo mediante el que se produce un apoyo efectivo a más de 250 alumnos con TEA con la finalidad última de que reciban una educación inclusiva que les permita adquirir y/o potenciar sus capacidades, posibilitarles un adecuado desarrollo personal y contribuir en la mejora de su calidad de vida. Por consiguiente, estas organizaciones han de ofrecer servicios de calidad como uno de sus valores intrínsecos, de forma que respondan tanto a las necesidades como a las expectativas de las personas con discapacidad, quedando sus destinatarios satisfechos con el servicio recibido (BarrancoExpósito, 2002; CARM, 2003).

En este contexto, el objetivo de este trabajo es evaluar la calidad percibida y la satisfacción de los usuarios del Servicio de Apoyo Educativo de ASTRADE. Ahora bien, al analizar la calidad de un servicio hay que tener presente que su medición se realiza a través de las percepciones, de ahí que sea tan importante prestar un servicio de calidad como que así lo perciban sus usuarios (Grönroos, 1994). Además, debido a los efectos del TEA y/o a la edad de los usuarios, serán los familiares o tutores quienes ejercerán de portavoces de dichas percepciones, lo que permitirá descubrir los niveles de calidad percibida y de satisfacción que se desean medir (García et al., 2008).

Para cumplir con los objetivos de esta investigación, el artículo se estructurará de la siguiente manera. En primer lugar se detallarán las características del Servicio de Apoyo Educativo prestado por ASTRADE. A continuación, se explicará la metodología empleada en el presente trabajo. En tercer lugar, se expondrán los resultados del estudio empírico y finalmente, las conclusiones obtenidas.

\footnotetext{
${ }^{4}$ Asociación para la atención de personas con Trastornos Generalizados del Desarrollo de la Región de Murcia.

${ }^{5}$ Federación de Organizaciones en favor de Personas con Discapacidad Intelectual y Parálisis Cerebral de la Región de Murcia.
} 


\subsection{Características del servicio}

El Servicio de Apoyo Educativo es el "tratamiento clave" que desarrolla ASTRADE, siendo sus objetivos principales mejorar el apoyo educativo y psicopedagógico del alumnado con TEA a través del desarrollo de programas de logopedia, apoyo pedagógico, y desarrollo de la autonomía personal y social. Ello se logra mediante una adecuada coordinación entre los servicios educativos de la Consejería de Educación, Universidades y Empleo de la CARM y los profesionales de ASTRADE.

En mayo de 2014, un grupo de 59 terapeutas cualificados prestan este servicio a 265 usuarios repartidos en 127 centros educativos de la CARM, abarcando toda la formación reglada no universitaria, tanto en centros concertados como públicos, tanto en aulas abiertas como en cualquier modalidad educativa: combinada, educación especial e integración.

Los destinatarios del programa son todos los residentes de la Región de Murcia y que estén diagnosticados en alguna de las categorías del TEA (Asociación Americana de Psiquiatría, 2013): Trastorno autista, Trastorno desintegrativo de la infancia, Síndrome de Asperger, y Trastorno Generalizado del Desarrollo no especificado.

Este servicio se desarrolla coincidiendo con el calendario y horario escolar de los centros educativos, centrando su intervención en dos ámbitos bien diferenciados: el educativo y el familiar.

En el ámbito educativo, el terapeuta realiza un apoyo individualizado al alumno en el contexto natural, actuando como modelo y mediador en la comunicación y la interacción social entre las personas con autismo y las personas de su entorno (el tutor, los demás compañeros, otros profesionales, etc.), para favorecer y potenciar la adaptación e integración del alumno en diferentes situaciones educativas (Millá y Mulas, 2009), así como el desarrollo de todas sus capacidades, teniendo como objetivo primordial lograr la inclusión de las personas con TEA en la sociedad. El terapeuta, además, colabora y coordina con el tutor y el equipo de apoyo del centro educativo la realización de distintas actividades, entre las que se encuentran la elaboración de adaptaciones curriculares en los contenidos y en la metodología, las evaluaciones, el material específico para el niño y para los profesionales que trabajan con él, las orientaciones concretas sobre unidades didácticas y la coordinación con la familia.

Por su parte, la intervención en el ámbito familiar (Rodríguez-Torrens, 2006) consiste en apoyar psíquica y emocionalmente a las familias, sobre todo en el momento inicial de diagnóstico, además de orientar a los padres ante determinados problemas de alimentación, comunicación, conducta, autonomía, sueño, etc., así como de hacer un seguimiento de los hermanos recién nacidos. Los profesionales de la Asociación son conscientes del gran deterioro que sufren las familias, tanto en el momento inicial como a lo largo de toda la vida del hijo con TEA (Martínez-Martín y Bilbao-León, 2008). 
Las horas de tratamiento que recibe cada usuario varían entre una y diez por semana, y dependen de la severidad del trastorno y de la capacidad económica de la familia. Es necesario señalar que, a pesar de contar con subvenciones por parte de la CARM, tanto la reducción de las mismas como el alto coste del tratamiento, debido a la atención individualizada y muy específica para cada usuario, provocan que las familias tengan que soportar una cuantía importante del coste del servicio. En los casos en los que solo se contrata una hora semanal, no se realiza intervención con el usuario, sino seguimiento de su evolución y coordinación con los profesionales del centro educativo. Sin embargo, más de diez horas de tratamiento a la semana indicarían la conveniencia de su inclusión en un centro especial, pudiendo en este caso ser usuario del servicio educativo en la modalidad de seguimiento. Actualmente, la media de horas que reciben semanalmente los usuarios del servicio educativo es de 4,5.

De la prestación del servicio conviene destacar la realización de diversos informes a lo largo del curso escolar, como son los programas de intervención, los informes de evolución y los informes de evaluación. Por un lado, el programa de intervención lo realiza el terapeuta durante las primeras semanas del curso escolar, siguiendo el Inventario del Espectro Autista (Rivière, 2002), también conocido como IDEA, donde se recogen los objetivos que se pretenden alcanzar con cada usuario del servicio a lo largo del curso. Por su parte, el informe de evolución lo realizan los responsables del equipo técnico a petición de la familia o de algún organismo oficial en el transcurso del tratamiento, y los terapeutas colaboran en su realización aportando la información que les solicite su coordinadora. Por último, el informe de evaluación se cumplimenta al finalizar el tratamiento o curso académico, y se devuelve a los padres y a los centros educativos durante la última semana del curso escolar.

\section{OBJETIVOS}

\subsection{Objetivo general}

El objetivo general de este trabajo consiste en evaluar el nivel de calidad percibida y de satisfacción de los usuarios del Servicio de Apoyo Educativo de ASTRADE.

\subsection{Objetivos específicos}

Los objetivos específicos que se pretenden conseguir con este trabajo pueden desglosarse en los siguientes:

a) Analizar el nivel de calidad percibida por los usuarios en relación al Servicio de Apoyo Educativo de ASTRADE.

b) Conocer la satisfacción de los usuarios con el Servicio de Apoyo Educativo de ASTRADE, a nivel específico y a nivel general.

c) Estudiar las dimensiones que configuran la calidad de servicio según el modelo SERVQUAL, para conocer cómo valoran los usuarios los distintos elementos que inciden en la calidad del servicio prestado. 


\section{METODOLOGÍA}

\subsection{Muestra}

La población objeto de estudio son todos los usuarios del Servicio de Apoyo Educativo de ASTRADE, sobre los que se realizaron 127 encuestas válidas (48,47\% de la población total), lo que supone un error muestral del $6,25 \%$ con un nivel de confianza del 95\% (Tabla 1).

Tabla 1. Ficha técnica del estudio.

\begin{tabular}{|l|l|}
\hline \multicolumn{1}{|c|}{ Tipo de encuesta realizada } & \multicolumn{1}{c|}{ Cuestionario estructurado } \\
\hline Lugar de realización de la encuesta & Domicilio usuario, centro educativo \\
\hline Universo & $\begin{array}{l}\text { Usuarios del Servicio de Apoyo Educativo de } \\
\text { ASTRADE. 262 niños (Mayo 2013) }\end{array}$ \\
\hline Tamaño de la Muestra & 127 encuestas válidas \\
\hline Error muestral & $\pm 6,25 \%$ \\
\hline Nivel de confianza & $95 \%(\mathrm{p}=\mathrm{q}=0,5)$ \\
\hline Fecha del trabajo de campo & Mayo-Junio 2013 \\
\hline
\end{tabular}

Fuente: Elaboración propia.

De los 127 hijos de los encuestados, el $82,7 \%$ son niños y el resto niñas, una cifra muy similar a la prevalencia del autismo por sexo donde, de cada cinco afectados, cuatro suelen ser varones (Díaz-Atienza, García-de-Pablos y Martín-Romera, 2004). Más de la mitad $(52,4 \%)$ se encontraban recibiendo este servicio por un periodo superior a seis años, es decir, sus familiares lo contrataron en 2007 o con anterioridad. Por lo tanto, gran parte de los encuestados cuenta con una amplia experiencia, siendo su valoración del servicio de gran valor para la Asociación. Destacar también que un $11,8 \%$ de los familiares contrató este servicio durante 2013, en un contexto económico poco favorable.

Un total de 17 niños presenta más del $75 \%$ de minusvalía, estando en el otro extremo unos 11 niños con un grado inferior al 33\%. La mayoría de los niños (59), tiene un grado de minusvalía en el intervalo de $33 \%$ a $50 \%, 15$ en el intervalo de $51 \%$ a $64 \%$ y 18 en el intervalo de $65 \%$ a $74 \%$. Por último, 7 de ellos no registran minusvalía o ésta se encuentra en trámite.

En relación al curso escolar del niño, el 56,7\% está cursando primaria, el 18,1\% infantil y el $15 \%$ secundaria. A más distancia se encuentran los matriculados en bachiller $(3,9 \%)$ y otros niveles $(6,3 \%)$.

\subsection{Instrumentos}

Para cumplir con los objetivos de este trabajo se han realizado dos estudios empíricos con fuentes primarias, entrevistas personales y encuestas. Con el primero se pretende obtener una visión más profunda del funcionamiento de la Asociación y de los servicios que presta. Con la encuesta se persigue conseguir información de la percepción de dichos servicios por parte de los asociados. 
Las entrevistas se han realizado a responsables, coordinadores y terapeutas de la Asociación, en las que se trataron aspectos generales sobre la Asociación, los servicios prestados, el Servicio de Apoyo Educativo, las instalaciones, y temas específicos de las personas con TEA. La información obtenida fruto de las entrevistas realizadas se ha incorporado al epígrafe 2 de este trabajo, al tiempo que confirman los resultados obtenidos en la investigación cuantitativa.

Por su parte, la encuesta se ha realizado mediante cuestionario estructurado, que se compone de varios bloques de preguntas. El primer bloque se refiere a los datos de clasificación de los usuarios, como el sexo, la edad, el nivel de estudios y el grado de minusvalía, los cuales han sido comentados en el apartado anterior. El siguiente bloque se refiere a la calidad percibida. Para su medición se utilizó el instrumento SERVQUAL de Parasuraman, Zeithaml y Berry (1988), aplicado en diferentes contextos (Asad et al., 2013; Cabello y Chirinos, 2012; Campos-Soto, 2013; Lee y Hing, 1995; Medina-Tornero y Medina-Ruiz, 2011; Saleh y Ryan, 1991), lo que demuestra su contrastada validez. Este modelo se compone de 22 ítems agrupados en cinco dimensiones: Aspectos Tangibles, Fiabilidad, Sensibilidad, Seguridad, y Empatía. La primera dimensión, Aspectos Tangibles, se refiere a lo que es visible por el usuario, como las instalaciones, los equipos, los empleados y los materiales de la empresa que presta el servicio. La segunda dimensión, Fiabilidad, abarca cuestiones sobre tiempos de respuesta y realización correcta del servicio. La tercera dimensión, Sensibilidad, se refiere a la voluntad de los empleados en ayudar a los usuarios del servicio. La cuarta dimensión, Seguridad, se refiere a la confianza, a la amabilidad y a los conocimientos o aptitudes de los empleados de la organización. La quinta y última dimensión, Empatía, mide la atención individualizada y el grado en el que la institución y sus empleados se preocupan por los intereses de sus usuarios y comprenden sus necesidades. Los encuestados valoran cada uno de estos ítems mediante una escala tipo Likert de siete puntos, desde (1) "totalmente en desacuerdo" hasta (7) "totalmente de acuerdo". Con la respuesta a estas preguntas, la organización conocerá sobre qué elementos de los que intervienen en la calidad del servicio es preciso actuar para mejorar el servicio prestado.

El siguiente instrumento utilizado es la escala de Satisfacción con el servicio, desarrollada por Mano y Oliver (1993) y Oliver (1997), que hemos adaptado para evaluar el grado de satisfacción con el resultado de un servicio. Consta de 12 ítems, es de tipo Likert y tiene siete puntos, desde (1) "totalmente en desacuerdo" hasta (7) "totalmente de acuerdo". Este instrumento también ha sido ampliamente utilizado por la literatura sobre satisfacción del consumidor y del usuario (Parra-Meroño, 2006), demostrándose su validez general. Las cuestiones que recoge esta escala se relacionan, por un lado, con la contratación del servicio y, por otro, con la persona que lo recibe. En lo que se refiere a la contratación, se pregunta si el servicio es uno de los mejores que se podrían haber contratado, si fue una sabia decisión y están seguros de que acertaron al elegir el servicio, si funciona como esperaba, y si están contentos y satisfechos con la contratación del mismo. En cuanto al destinatario del servicio, se pregunta si el servicio es exactamente el que necesitaba su hijo y le supone una buena experiencia.

El tercer instrumento aplicado es la escala de Satisfacción general (Crosby y Stephens, 1987), adaptada por Parra (2006), cuyos autores la aplicaron 
originalmente para medir la intensidad de la satisfacción con la prestación de servicios y se centra en los aspectos emocionales de la satisfacción, por lo que algunos autores la denominan escala de agrado (Andreu, 2002). Nuestra adaptación se compone de tres ítems, es de tipo Likert y tiene siete puntos. De igual manera que la escala de Mano y Oliver (1993), este instrumento presenta una contrastada fiabilidad y validez, tanto en su estudio original como en muchos otros que la han adaptado y/o aplicado (Eroglu y Machleit, 1990). Este instrumento formula tres preguntas con la finalidad de averiguar si el usuario que recibe un determinado servicio está, en general, satisfecho, contento y a favor del mismo.

\subsection{Procedimiento}

El cuestionario se hizo llegar a los familiares mediante correo electrónico, correo postal y en persona en las distintas sedes de la Asociación. De las 127 encuestas que realizaron los familiares, 94 fueron cumplimentadas en sus domicilios, 26 en persona y 7 vía telefónica. Los resultados fueron analizados con el software de análisis estadístico SPSS 21.

\section{RESULTADOS}

En este epígrafe se describen los resultados alcanzados en el estudio empírico. En primer lugar, se presentan los referidos a la fiabilidad de los instrumentos de medida empleados. En segundo lugar, se detallan los estadísticos descriptivos para cada una de las escalas utilizadas y, en el caso de SERVQUAL, se muestra también esta serie de datos para sus cinco dimensiones. En tercer lugar, se comentan los elementos de la calidad percibida más valorados por los usuarios. En cuarto y último lugar, se presentan las correlaciones entre los distintos instrumentos de medida.

La fiabilidad de las tres escalas es buena, con unos valores del Alfa de Cronbach (George y Mallery, 2003) superiores a 0,8 en los tres instrumentos, y mayor de 0,9 en las escalas SERVQUAL y Satisfacción con el servicio. Además, los resultados del análisis muestran una alta fiabilidad para cada una de las cinco dimensiones de las que se compone la escala SERVQUAL (Tabla 2).

Tabla 2. Análisis de Fiabilidad y Estadísticos descriptivos.

\begin{tabular}{|l|c|c|c|c|r|}
\hline \multicolumn{1}{|c|}{ Escala } & İtems & $\begin{array}{c}\text { a de Cronbach } \\
\text { tipificada }\end{array}$ & M & DT & \multicolumn{1}{c|}{ ANOVA } \\
\hline Servqual & 22 & 0,952 & 6,336 & 0,399 & $\mathrm{~F}=31,522$ \\
\hline Aspectos tangibles & 4 & 0,786 & 5,915 & 0,524 & $\mathrm{~F}=53,684$ \\
\hline Fiabilidad & 5 & 0,919 & 6,250 & 0,134 & $\mathrm{~F}=7,639$ \\
\hline Sensibilidad & 4 & 0,864 & 6,537 & 0,170 & $\mathrm{~F}=14,672$ \\
\hline Seguridad & 4 & 0,874 & 6,594 & 0,148 & $\mathrm{~F}=15,338$ \\
\hline Empatía & 5 & 0,852 & 6,392 & 0,202 & $\mathrm{~F}=12,390$ \\
\hline Satisfacción con el servicio & 12 & 0,947 & 6,407 & 0,118 & $\mathrm{~F}=4,699$ \\
\hline Satisfacción general & 3 & 0,814 & 6,404 & 0,217 & $\mathrm{~F}=25,176$ \\
\hline
\end{tabular}

Fuente: Elaboración propia. 
Los resultados obtenidos, sobre un máximo de siete puntos, reflejan una valoración muy positiva del Servicio de Apoyo Educativo. Los usuarios perciben un servicio de calidad y declaran estar satisfechos tanto con los resultados del mismo como a nivel general. A continuación, se comentan algunos de los aspectos que inciden en la calidad del servicio según el instrumento SERVQUAL, y que, por las características del servicio prestado, resulta conveniente destacar.

En primer lugar, en cuanto a la dimensión "Fiabilidad", los familiares perciben que la organización muestra un sincero interés en solucionar los problemas $(6,33)$ que se puedan presentar, de forma rápida y eficaz $(6,14)$, realizando el servicio de forma correcta a la primera $(6,11)$, en el plazo de tiempo en el que se han comprometido $(6,24)$, tratando de cometer los menores errores posibles $(6,43)$. En segundo lugar, los resultados obtenidos en la dimensión "Sensibilidad" ponen de relieve que los terapeutas informan con precisión sobre la evolución del servicio prestado $(6,46)$, y que además nunca están demasiado ocupados para resolver las dudas de los padres $(6,61)$, estando siempre dispuestos a ayudarles $(6,73)$. En tercer lugar, la dimensión "Seguridad" refleja que los trabajadores del Servicio de Apoyo Educativo transmiten a los asociados altos niveles de confianza $(6,60)$ y amabilidad $(6,80)$, además de sentirse seguros en el desarrollo del servicio $(6,50)$, que es prestado por trabajadores cualificados capaces de resolver las dudas que puedan surgir $(6,47)$. En cuarto lugar, la dimensión "Empatía" refleja que los padres perciben por parte de la Asociación una atención personal $(6,65)$ e individualizada $(6,54)$, así como que los trabajadores comprenden las necesidades específicas de las familias $(6,34)$ y se preocupan por sus intereses $(6,26)$. Esta dimensión es especialmente valorada en los estudios realizados sobre personas con discapacidad intelectual (Potocnik, Moliner y Martínez-Tur, 1997; TamaritCuadrado, 2002), e incluso algunos autores (Martínez-Martín y Bilbao-León, 2008) señalan la empatía como un factor clave en los programas de intervención. En quinto y último lugar, los asociados perciben que los "Aspectos tangibles" de los que dispone la organización son de calidad. Así, las instalaciones donde se realiza el servicio educativo son visualmente atractivas $(5,33)$, los empleados del servicio tienen una apariencia pulcra $(6,61)$, los equipos utilizados en el desarrollo del servicio aparentan ser modernos $(5,85)$ y los materiales relacionados con el servicio, tales como folletos, pictogramas o agendas, resultan igualmente atractivos.

En cuanto a la satisfacción, los familiares manifiestan un alto grado de satisfacción con el resultado del servicio. En concreto, los padres consideran que es uno de los mejores servicios que podrían haber contratado para su hijo $(6,39)$, que fue una sabia decisión $(6,51)$ y que funciona como esperaban $(6,46)$. Además, el servicio prestado es exactamente el que necesitaba su hijo $(6,20)$, al que le supone una buena experiencia $(6,39)$. Por su parte, los asociados también se muestran satisfechos $(6,29)$, contentos $(6,27)$ y a favor $(6,65)$ del servicio que reciben.

\section{CONCLUSIONES Y DISCUSIÓN}

La valoración de los usuarios del servicio ha sido muy positiva, resultados que ponen de relieve que la Asociación ha conseguido que sus usuarios perciban altos niveles de calidad (Medina-Tornero y Medina-Ruiz, 2011) y, además, estén muy satisfechos 
con el servicio. Consideramos que estos resultados se deben a una serie de factores que son clave en las organizaciones prestatarias de este tipo de servicios.

Por un lado, los familiares son conscientes de la gran dificultad que supone realizar el trabajo con personas con TEA y, por lo tanto, de la importancia de una gran implicación e interés hacia estas personas, y el apoyo a sus familiares, para favorecer una mejora en su desarrollo personal (Millá y Mulas, 2009). Como dice Tamarit-Cuadrado (2005, p.185), los profesionales que prestan el servicio de apoyo educativo han de ser "excelentes personas desde la ética y la empatía", ya que la percepción de satisfacción con un servicio depende, en gran medida, de la persona que lo presta.

Por otro, los terapeutas están enormemente concienciados sobre los problemas que preocupan a los padres y la enorme ansiedad e inquietudes que una falta de respuestas puede provocarles (Martínez-Martín y Bilbao-León, 2008; BenitesMorales, 2010), motivo por el que los profesionales de la Asociación transmiten estar siempre dispuestos a ayudar a las familias, asesorándolas y ofreciéndoles una rápida y eficaz respuesta a las dudas que se les puedan plantear.

Además, a pesar de que el trastorno autista se caracteriza por la dificultad en el aprendizaje (Benites-Morales, 2010) y por la lentitud en los avances del tratamiento, los familiares son conscientes de ello y valoran de forma muy positiva el desarrollo en el tiempo adecuado.

Por último, el modelo de intervención educativa de la Asociación ha demostrado ser adecuado para sus destinatarios así como favorecer el desarrollo educativo y personal de los usuarios. Por tanto, se estima que es necesario que las organizaciones del sector apuesten por la mejora de aquellos aspectos que inciden en el desarrollo personal y la calidad de vida de las personas con TEA, con el objetivo más que razonable de conseguir la plena inclusión de estas personas en la sociedad.

\section{REFERENCIAS}

Andreu, L. (2002). Análisis de la satisfacción en la experiencia del consumidor: una aplicación en empresas de servicios de ocio y turismo. Investigaciones Europeas de Dirección y Economía de la Empresa, 8(3), 199-214.

Artigas, J. (1999). El lenguaje en los trastornos autistas. Revista de neurología, 28(2), 118-23.

Asad, I., Hammad, N., Fida, H., Muhammad, R. M., Saleha, M. \& Zahoor, S. (2013). Evaluating Business Schools Service Quality Using SERVQUAL Model. Journal of Basic and Applied Scientific Research, 3(5), 710-716.

Asociación Americana de Psiquiatría (APA). (2013). Manual diagnóstico y estadístico de los trastornos mentales, Quinta Edición (DSM-5).

Barranco-Expósito, C. (2002). Calidad del servicio: Estudio realizado en CAMPs de Tenerife. Psychosocial Intervention, 11(3), 371-382. 
Benites-Morales, L. (2010). Autismo, familia y calidad de vida. Cultura, 24, 1-20.

Cabello, E. \& Chirinos, J. L. (2012). Validación y aplicabilidad de encuestas SERVQUAL modificadas para medir la satisfacción de usuarios externos en servicios de salud. Revista Médica Herediana, 23(2), 88-95.

Campos-Soto, R. (2013). Calidad de servicio percibido según modelo Servqual, del Hospital Santa María del Socorro, y su relación con la satisfacción de los pacientes, 2008. Revista Enfermería a la Vanguardia, 1(1), 16.

Comunidad Autónoma de la Región de Murcia (CARM). (2003). Sistema de Servicios Sociales de la Región de Murcia, 10 de abril de 2003, Ley 3/2003, art.42.

Crosby, L. A. \& Stephens, N. (1987). Effects of Relationship Marketing on Satisfaction, Retention, and Prices in the Life Insurance Industry. Journal of Marketing Research, XXIV(November), 404-411.

Crosso, C. (2010). El derecho a la educación de personas con discapacidad: impulsando el concepto de educación inclusiva. Revista Latinoamericana de Educación Inclusiva, 4(2), 79-95.

Cuesta-Gómez, J. L., Casado-Muñoz, R., y Lezcano-Barbero, F. (2010). Evaluación de la calidad de vida en personas con Trastornos del Espectro Autista. VERTEX, Revista Argentina de Psiquiatría, XXI, 117-125.

Díaz-Atienza, F., García-de-Pablos, C. \& Martín-Romera, A. (2004). Diagnóstico precoz de los Trastornos Generalizados del Desarrollo. Revista de Psiquiatría y Psicología del Niño y del Adolescente, 4(2), 127-144.

Eroglu, S. A. \& Machleit, K. (1990). An Empirical Study of Retail Crowding: Antecedents and Consequences. Journal of Retailing, 66(2), 201-221.

Fernández-Jaén, A., Martín Fernández-Mayoralas, D., Calleja-Pérez, B., \& MuñozJareño, N. (2007). Síndrome de Asperger: diagnóstico y tratamiento. Revista de Neurología, 44(2), 53-55.

Fortea-Sevilla, M.D.S., Escandell-Bermúdez, M. O., \& Castro-Sánchez, J. J. (2013). Estimación de la prevalencia de los trastornos del espectro autista en Canarias. Anales de Pediatría, 79(6), 352-359.

García, F. A., Mirete, A., Marín, C. \& Romero, L. (2008). Satisfacción del cliente familia en atención temprana: valoración de la importancia que otorgan a distintos aspectos del servicio. Siglo Cero, 39(227), 55-74.

García-Dávila, N., \& Crespo-Cuadrado, M. (2008). Caminando hacia la calidad de vida. Siglo Cero, 39(227), 35-54.

George, D., \& Mallery, P. (2003). SPSS for Windows step by step: A simple guide and reference. 11.0 update (4th ed.). Boston: Allyn \& Bacon. 
Giné, C., \& Durán, D. (2010). Progresando hacia la Educación Inclusiva. Boletín del Real Patronato sobre Discapacidad, (69), 10-13.

Grönroos, C. (1994). Marketing y gestión de servicios: la gestión de los momentos de la verdad y la competencia en los servicios. Madrid: Díaz de Santos.

Lee, Y. L. \& Hing, N. (1995). Measuring quality in restaurant operations: an application of the SERVQUAL instrument. International Journal of Hospitality Management, 14(3), 293-310.

Mano, H. \& Oliver, R. L. (1993). Assessing the dimensionality and structure of the consumption experience: evaluation, feeling, and satisfaction. Journal of Consumer research, 20(December), 451-466.

Martínez-Martín, M. Á., \& Bilbao-León, M. C. (2008). Acercamiento a la realidad de las familias de personas con autismo. Intervención Psicosocial, 17(2), 215-230.

Medina-Tornero, M. \& Medina-Ruiz, E. (2011). Análisis de la calidad percibida en usuarios/as de servicios sociales comunitarios. Zerbitzuan: Gizarte zerbitzuetarako aldizkaria=Revista de servicios sociales, (50), 85-100.

Millá, M. G., \& Mulas, F. (2009). Atención temprana y programas de intervención específica en el trastorno del espectro autista. Revista de Neurología, 48(2), 47-52.

Oliver, R. L. (1997). Satisfaction: A Behavioral Perspective on the Consumer. Nueva York: McGraw-Hill.

Parasuraman, A., Zeithaml, V. A. \& Berry, L. L. (1988). Servqual. Journal of retailing, 64(1), 12-40.

Parra-Meroño, M. C. (2006). La Insatisfacción del Consumidor y las Respuestas ante la Insatisfacción: un Análisis Teórico y Empírico. (Tesis Doctoral). Murcia: Universidad Católica de Murcia.

Potocnik, K., Moliner, C., \& Martínez-Tur, V. (1997). ¿Influye el tipo de servicio en las evaluaciones de los usuarios? Servicios comerciales vs. sin ánimo de lucro. Revista Electrónica de Motivación y Emoción. Monográfico de La Motivación Del Consumidor: Estudios Experimentales, XIII(35-36), 139-153.

Rivière, A. (2002). IDEA: inventario de espectro autista. Buenos Aires: Fundec.

Rodríguez-Torrens, E. (2006). Atención a familias en los servicios para personas con Trastornos del Espectro Autista. Educación y Futuro, 14, 109-130.

Saleh, F. \& Ryan, C. (1991). Analysing service quality in the hospitality industry using the SERVQUAL model. Service Industries Journal, 11(3), 324-345. 
Schalock, R. I., \& Verdugo-Alonso, M. Á. (2007). El concepto de calidad de vida en los servicios y apoyos para personas con discapacidad intelectual. Siglo Cero, 38(224), 21-36.

Tamarit-Cuadrado, J. (2002). Calidad en los servicios para personas con discapacidad intelectual en el ámbito europeo. Documentación social: Revista de Estudios Sociales y Sociología Aplicada, (128), 241-254.

Tamarit-Cuadrado, J. (2005). Autismo: modelos educativos para una vida de calidad. Revista de Neurología, 40(1), 181-186. 